\title{
NEUROINFLAMMATORY EFFECTS OF TDCS IN OVARIECTOMIZED RATS WITH CHRONIC INFLAMMATION
}

Clin Biomed Res. 2021;41(4):332-338 1 Laboratório de Farmacologia da Dor e Neuromodulação: Investigações Pré-Clínicas, Centro de Pesquisa Experimental, Hospital de Clínicas de Porto Alegre. Porto Alegre, RS, Brasil.

2 Programa de Pós-Graduação em Medicina: Ciências Médicas, Universidade Federal do Rio Grande do Sul. Porto Alegre, RS, Brasil.

3 Programa de Pós-Graduação em Saúde e Desenvolvimento Humano, Universidade La Salle. Canoas, RS, Brasil.

4 Faculdade de Medicina, Universidade Federal do Pará. Belém, PA, Brasil.

5 Programa de Pós-Graduação em Odontologia, Universidade Federal do Rio Grande do Sul. Porto Alegre, RS, Brasil.

Corresponding author: Iraci Lucena da Silva Torres iltorres@hcpa.edu.br Laboratório de Farmacologia da Dor e Neuromodulação: Investigações Pré-Clínicas, Centro de Pesquisa Experimental, Hospital de Clínicas de Porto Alegre

Rua Ramiro Barcelos, 2350 900035-070, Porto Alegre, RS, Brasil.
Iraci L. S. Torres ${ }^{1,2}$, Carla de Oliveira ${ }^{1,2}$, Vanessa Leal Scarabelot ${ }^{1}$, Rafael Vercelino ${ }^{1}$, Andressa de Souza ${ }^{1,3}$, Sônia Fátima Silva Moreira ${ }^{1,2,4}$, Fernanda Visioli ${ }^{5}$, Wolnei Caumo ${ }^{1,2}$, Liciane Fernandes Medeiros ${ }^{1,3}$

\section{ABSTRACT}

Introduction: Postmenopausal women are more susceptible to chronic conditions, such as osteoporosis, arthritis, and other inflammatory diseases. We investigated the effects of transcranial direct current stimulation (tDCS) on biomarker levels in ovariectomized rats subjected to an inflammatory model.

Methods: Twenty adult female Wistar rats underwent ovariectomy and complete Freund's adjuvant (CFA)-induced inflammation. We divided them into 2 groups: OAS (sham tDCS) and OAT (active tDCS). Fifteen days later, the rats underwent bimodal tDCS treatment (20 min, $0.5 \mathrm{~mA}, 8$ days). After $24 \mathrm{~h}$ of the last tDCS session, we killed the rats and collected tissue samples (hypothalamus, cerebral cortex, and brainstem) for biomarker analysis by ELISA. We removed the paws for histological analysis.

Results: Active tDCS increased hypothalamic and cortical TNF- $\alpha$ and NGF levels, hypothalamic and brainstem IL-1 $\beta$ levels, and hypothalamic IL-10 levels. Histology of paws showed an inflammatory profile. We observed a small tDCS effect, not statistically significant.

Discussion: Bimodal tDCS had an effect on the central inflammatory axis, with a small effect on the peripheral site as evaluated by histology in the current study.

Keywords: Inflammation; Cytokines; Estrogen; Ovariectomy; tDCS

\section{INTRODUCTION}

Menopause is the age-related loss of ovarian function and represents a state of profound estrogen deprivation ${ }^{1}$. It could be responsible, in part, for the elevation of pro-inflammatory cytokines seen in women of advanced age $^{2}$. Postmenopausal women are more susceptible to chronic conditions, such as osteoporosis, arthritis, and other inflammatory diseases, which are associated with changes in the expression and secretion of pro-inflammatory cytokines ${ }^{3}$. Also, cognitive impairments are common during menopause, which is marked by a decrease in hormone levels ${ }^{4}$.

Interestingly, estrogen deficiency may result in a significant increase in inflammatory factors in the blood, such as tumor necrosis factor-alpha (TNF- $\alpha$ ) and interleukin-1 beta (IL-1 $\beta)^{5}$. TNF- $\alpha$ levels increase abruptly after 51 years of age $^{6}$, which is the average age of menopause. Moreover, older postmenopausal women often present with suppressed nerve growth factor (NGF) expression ${ }^{7}$ and significantly decreased serum IL-10 levels ${ }^{8}$. It is important to point out that they show greater upregulation of the genes involved in inflammation and immune function than older men ${ }^{9,10}$.

Changes observed during menopause may also affect central nervous system structures related to hormones and inflammatory systems. The hypothalamus is a part of the brain that controls body temperature and may be involved in hot flashes (HFs) by increasing gonadotropin-releasing hormone secretion ${ }^{11}$. 
Also, increased brainstem activity has been detected before the detectable onset of HFs by using functional magnetic resonance imaging ${ }^{12}$, and insular and prefrontal activity appeared following HF onset. The menopausal transition period has pronounced effects on the human brain structure, connectivity, and energy metabolism, which is related to an adaptive process serving the transition into later life.

Preclinical studies suggest that ovariectomy affects inflammatory functions under basal conditions, but little is known about the effects of ovariectomy on inflammatory responses induced under immune stress conditions ${ }^{13}$. In female rats, the potential effect of ovariectomy on inflammatory pain has not been elucidated. It is important to highlight that, in ovariectomized female rats, only high concentrations of formalin can demonstrate inflammatory and nociceptive responses ${ }^{14-16}$.

Transcranial direct current stimulation (tDCS), a technique that non-focally modulates plastic changes induced by pain-related neural networks, can be a useful tool in menopause management. A previous study by our research group showed both immediate and long-lasting antinociceptive effects of repeated sessions of bicephalic tDCS in a rat model of chronic inflammation ${ }^{17}$. In another study by our group, ovariectomized rats showed a hypernociceptive response associated with changes in peripheral and central brain-derived neurotrophic factor (BDNF) levels; cathodal tDCS treatment partially reversed the mechanical hyperalgesia response and completely reversed the decreased cortical BDNF levels ${ }^{18}$.

In this context, it is important to develop new studies on the effects of tDCS on inflammatory disease associated with low estrogen levels. Therefore, considering the need to develop novel strategies for menopause management, we investigated the effects of tDCS on central TNF- $\alpha, \mathrm{IL}-1 \beta, \mathrm{IL}-10$, and NGF levels in ovariectomized rats subjected to a model of chronic inflammation.

\section{METHODS}

\section{Animals}

We randomized 20 female Wistar rats (90 days old, 200-300 g) by weight and housed them in groups of 3 to 4 animals per polypropylene cage $(49 \mathrm{~cm} \times 34 \mathrm{~cm} \times 16 \mathrm{~cm})$ with sawdust-covered floor. We maintained all rats in a controlled environment $\left(22 \pm 2^{\circ} \mathrm{C}\right)$ under a standard light-dark cycle (lightson at 7 a.m. and lights-off at 7 p.m.), with water and chow (Nuvital, Porto Alegre, RS, Brazil) ad libitum. The institutional Animal Care and Use Committee (GPPG-HCPA protocol no. 2014.0262) approved all experiments and procedures, which were performed in accordance with the Guide for the Care and Use of Laboratory Animals, 8th ed. The experimental protocol complied with the ethical and methodological standards of the ARRIVE guidelines ${ }^{19}$.

\section{Experimental design}

We allowed the rats to acclimate to the study environment for 14 days before the beginning of the experiment and, subsequently, randomized them by weight. On day 1 , after performing ovariectomy with complete Freund's adjuvant (CFA)-induced inflammation via injection into the footpad under anesthesia, we divided the rats into the following 2 groups: OAS (treated with sham tDCS) and OAT (treated with active tDCS). Fifteen days later, the rats underwent daily tDCS sessions for 8 consecutive days. An experimenter blinded to group allocation performed biochemical and histological analyses.

\section{Surgical procedures}

We anesthetized the rats with $4 \%$ isoflurane for induction and $2 \%$ for maintenance and performed bilateral ovariectomy as described previously ${ }^{20}$. Surgery consisted of a dorsolateral transverse skin incision between the last rib and pelvis, followed by muscle dissection to expose the abdominal cavity. The ovary is in a fat pad beneath the muscles. We grasped the periovarian fat to lift and exteriorize the ovary. We crushed and ligated the fallopian tube, and then removed the ovary by cutting above the clamped area. We closed the muscles and skin incision with polyglactin and nylon sutures. We repeated the same procedure on the other side for bilateral ovariectomy. All rats received tramadol chlorhydrate ( $5 \mathrm{mg} / \mathrm{kg}$, i.p.) for postoperative pain relief.

\section{CFA-induced inflammation}

We purchased CFA from Sigma Chemical Co. (St Louis, MO, USA). The protocol used for CFAinduced inflammation is similar to previously described protocols ${ }^{21}$. Briefly, we anesthetized the rats with isoflurane and induced inflammation via a single $100 \mu \mathrm{L}$ intradermal injection of heat-killed Mycobacterium tuberculosis suspended in paraffin oil and mannide monooleate $(1 \mathrm{mg} / 1 \mathrm{~mL})$ into the right footpad.

\section{Vaginal smear}

Ten days after surgery, we obtained vaginal smears once daily to confirm hormonal status. We obtained and analyzed the samples as described previously ${ }^{22}$.

\section{Transcranial direct current stimulation (tDCS)}

After 14 days of CFA administration, the rats underwent a 20 min session of bicephalic tDCS every morning for 8 days, as described previously ${ }^{23,24}$.

\section{Tissue collection}

After $24 \mathrm{~h}$ of the last tDCS session, we killed the rats by decapitation and collected brain tissue samples 
(hypothalamus, cerebral cortex, and brainstem). We kept the samples frozen at $-80^{\circ} \mathrm{C}$ until analysis.

\section{Biochemical assays}

We determined TNF- $\alpha$, IL-1 $\beta$, IL-10, and NGF levels by sandwich ELISA using monoclonal antibodies specific for each measurement (R\&D Systems, Minneapolis, MN, USA). We measured total protein by Bradford's method using bovine serum albumin as standard and expressed the data as $\mathrm{pg} / \mathrm{mg}$ of protein.

\section{Histological scoring}

For histological analysis, we excised the hind paws and fixed them in $10 \%$ buffered formalin for 7 days. We then decalcified the paws with $10 \%$ nitric acid for $27 \mathrm{~h}$. We sectioned tissues and embedded them in paraffin. We prepared the slides by staining the tissue sections with hematoxylin and eosin. A pathologist blinded to treatment allocation performed a histological analysis of the tibiotarsal joint (ankle region). By optical microscopy, the pathologist graded the histological slides according to the percentage of infiltrating inflammatory cells compared with the surrounding tissue $0=$ absent; $1=$ mild $[1 \%-10 \%] ; 2=$ moderate $[11 \%-50 \%]$; and $3=$ severe $[51 \%-100 \%])$ and quantified the number of medium microscopic power fields (200x) occupied by the inflammatory process. We identified cells according to their morphological characteristics.

\section{Statistical analysis}

We expressed the data as mean and standard error of the mean (SEM). In biochemical assays, we used Student's t test for independent samples to compare the effects of tDCS. We analyzed the histological data using the Mann-Whitney test for independent samples. We considered differences to be statistically significant at $P<0.05$. We used SPSS 20.0 for statistical analysis.

\section{RESULTS}

\section{Assessment of hormonal status}

The vaginal smears of the ovariectomized rats presented an acyclical pattern exhibiting only the metestrus and diestrus stages, thus confirming the animal model of menopause ( $n=8-10$ per group, data not shown).

\section{Hypothalamic biomarker levels}

Active tDCS induced an increase in hypothalamic TNF- $\alpha$, IL-1 $\beta$, IL-10, and NGF levels (Student's t test, $P<0.01$ and $P<0.001$ for IL-10 and NGF; Figure 1A).

\section{Cerebral cortex biomarker levels}

Active tDCS induced an increase in cerebral cortex TNF- $\alpha$ and NGF levels (Student's t test, $P<0.05$ and $P<0.02$, respectively; Figure 1B), with no difference between the groups in IL-1 $\beta$ or IL-10 levels (Student's t test, $P>0.05$; Figure 1B).

\section{Brainstem biomarker levels}

Active tDCS induced an increase in brainstem IL-1 $\beta$ levels (Student's t test, $P<0.03$; Figure $1 \mathrm{C}$ ). There was no significant difference between the groups in TNF- $\alpha$, IL-10, or NGF levels (Student's t test, $P>0.05$; Figure 1C).

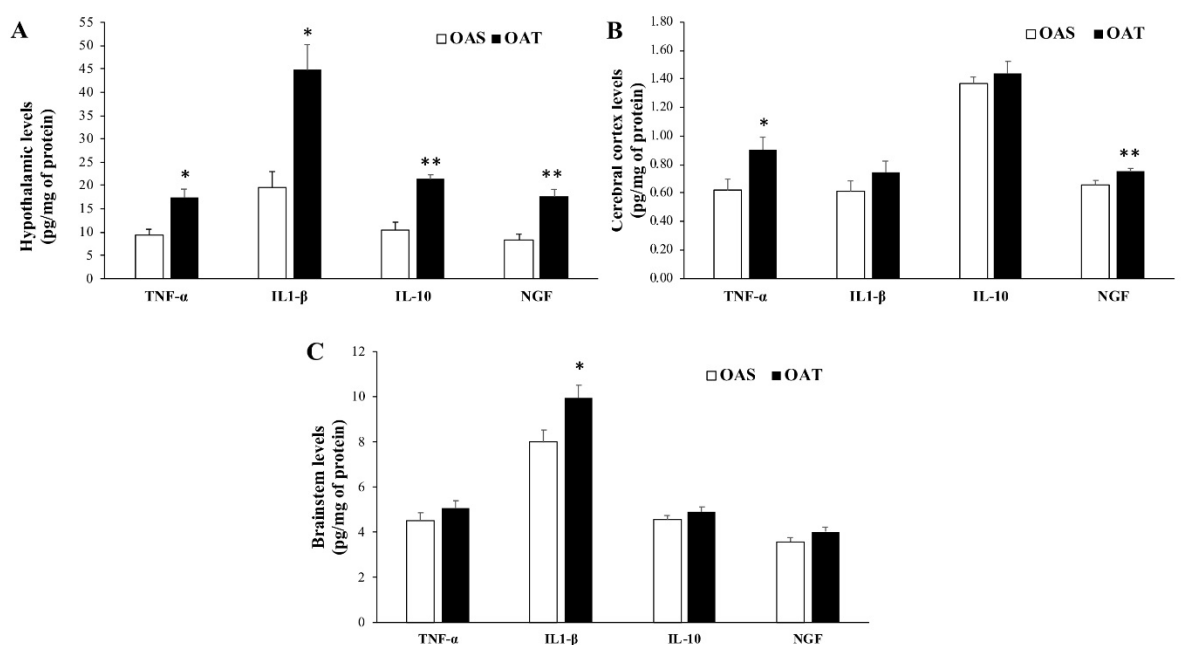

Figure 1: Biomarker levels in central nervous system (CNS) structures. Data are presented as mean \pm standard error of the mean (SEM) of pg/mg of protein. OAS (ovariectomized + CFA + sham tDCS) and OAT (ovariectomized + CFA + tDCS) ( $\mathrm{n}=9$ per group). A: Hypothalamic levels (Student's t test, ${ }^{*} P<0.01$ and ${ }^{* *} P<0.001$ ); B: Cerebral cortex levels (Student's t test, ${ }^{*} P<0.05$ and ${ }^{* *} P<0.02$ ); C: Brainstem levels (Student's t test, ${ }^{*} P<0.03$ ). 


\section{Histological analysis}

Figure 2 shows the histological findings for each group. We observed an inflammatory infiltrate of lymphocytes, plasmocytes, macrophages, and, more rarely, neutrophils in reaction to CFA injection in the subcutaneous tissues of the ankle joint. We also observed the formation of giant cells. The OAT group had lower inflammation scores, but there was no statistically significant difference in histological scoring between the groups (Mann-Whitney test, $P>0.05$; Table 1).

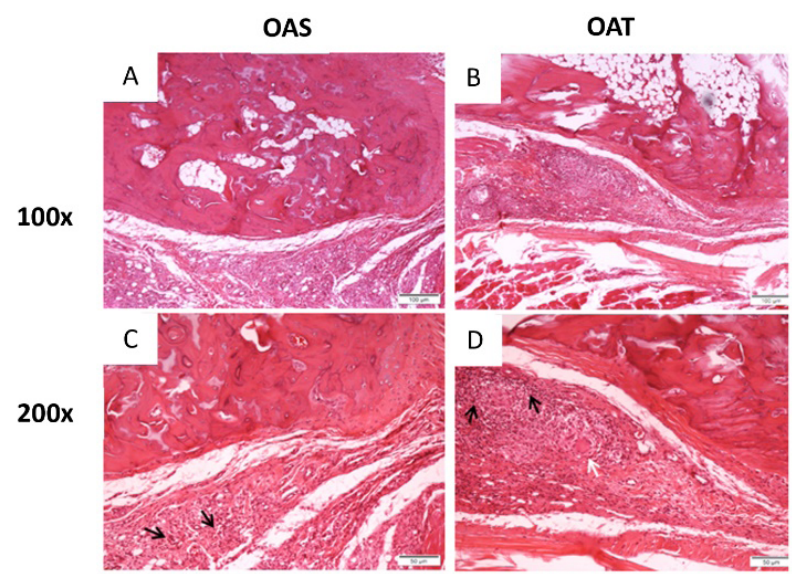

Figure 2: Hematoxylin and eosin-stained sections from the hind paws of rats (100x and $200 \times$ magnification) ( $n=3-5$ per group). A and C: OAS group showing inflammatory reaction with lymphocytes and plasma cells; $B$ and D: OAT group showing inflammatory reaction with mononuclear cells (black arrows) and macrophage aggregates (white arrow). There was no significant difference between the groups; Mann-Whitney test, $P>0.05$.

Table 1: Histological analysis of inflammatory processes in the tibiotarsal joint.

\begin{tabular}{lcccccc}
\hline Groups & OAS & \multicolumn{3}{c}{ OAT } & \\
\hline \multicolumn{5}{c}{ Median $(25 \%-75 \%)$} & Median $(25 \%-75 \%)$ & $P^{*}$ \\
$\begin{array}{l}\text { Histological } \\
\text { score }\end{array}$ & 3.000 & $(0.5-4)$ & 2.500 & $(0.25-4)$ & 0.9993 \\
$\begin{array}{l}\text { Microscopic } \\
\text { fields }\end{array}$ & 22.00 & $(0.5-29.5)$ & 15.00 & $(0-27)$ & 0.8053 \\
\hline $\begin{array}{l}\text { OAS: ovariectomized + } \\
\text { ovariectomized + CFA + tDCS. }\end{array}$ & + sFA & + & sham & tDCS; & OAT: \\
* No significant difference (Mann-Whitney test). & & \\
\end{tabular}

\section{DISCUSSION}

In the present study, our data demonstrated that ovariectomized rats with chronic inflammation treated with tDCS had increased levels of all investigated biomarkers: TNF- $\alpha$ in the hypothalamus and cerebral cortex; IL-1 $\beta$ in the hypothalamus and brainstem; and $\mathrm{IL}-10$ in the hypothalamus. In addition, tDCS induced an increase in NGF levels in the hypothalamus and cerebral cortex. It is important to note that tDCS-treated rats showed lower inflammation scores indicating a reduction in the number of inflammatory cells in the subcutaneous tissues of the ankle joint, although no statistical significance was reached.

Interestingly, a previous study highlighted that multiple sessions of tDCS triggered an inflammatory effect on the rat brain ${ }^{25}$. We showed that ovariectomy reduced hypothalamic and cerebral cortex BDNF levels, but tDCS reversed only the cerebral cortex levels ${ }^{18}$. Also, tDCS decreased hippocampal TNF levels in rats in a chronic pain model ${ }^{26}$. Moreover, bicephalic tDCS reduced IL-1 $\beta$ and IL-10 levels in the spinal cord and increased TNF- $\alpha$ levels in the cerebral cortex and spinal cord in rats subjected to neuropathic pain ${ }^{24}$.

In the current study, we observed an increase in hypothalamic IL-10 levels in tDCS-treated rats. IL-10 is a cytokine released during the resolution phase of inflammation that prevents tissue damage caused by infection and inflammation ${ }^{27,28}$. Therefore, we hypothesized that tDCS potentiates an inflammatory state in ovariectomized rats with chronic inflammation by modulating the neuroinflammatory pathway.

In neuromodulator analysis, we observed an increase in NGF levels in the hypothalamus and cerebral cortex of rats treated with bimodal tDCS. NGF can elicit the release of inflammatory mediators, as well as NGF itself, resulting in a positive feedback loop ${ }^{29,30}$. It also sensitizes adjacent nociceptive neurons in response to inflammation ${ }^{31}$. In our previous study of ovariectomized rats, cathodal tDCS had neuromodulatory effects on mechanical hyperalgesia and on the cortical levels of $\mathrm{BDNF}^{18}$, another neurotrophin involved in peripheral and central sensitization. It is noteworthy that tDCS is a neuromodulatory technique that induces potent neuroplastic changes, as observed in preclinical ${ }^{32}$ and clinical studies ${ }^{33}$. Although its mechanism is not fully understood, our findings highlight an interaction of the immune system with the neural circuitry in the model of ovariectomized rats with chronic inflammation promoted by tDCS.

There is growing evidence of a relationship between tDCS neuromodulation and immune system activation, and the effects of this technique are neuronally and non-neuronally driven ${ }^{34}$. Despite the changes in central biomarkers triggered by tDCS, in the peripheral analysis of the ankle joint, tDCS-treated rats had lower inflammation scores, that is, a reduced number of inflammatory cells, thus contributing with evidence on the mechanism of tDCS effects indexed by top-down activation ${ }^{35,36}$. It is important to note that this is the first study on 
tDCS treatment in ovariectomized rats subjected to chronic inflammation induced by CFA injection.

CFA-induced inflammation has been shown to increase the levels of cytokines and free radicals, and this "inflammatory soup" sensitizes nociceptive neurons, enhancing neuronal excitability, and leads to secondary hyperalgesia ${ }^{37}$. It should be noted that CFA-induced inflammatory response is biphasic, with an early (acute) and a late (chronic) phase ${ }^{38}$. Our study focused on the late phase of inflammation, which induces immunological events ${ }^{39}$, including an increase in the levels of inflammatory mediators, such as calcitonin gene-related peptide, NGF, IL-1 $\beta$, and TNF- $\alpha^{40}$.

Intraplantar administration of CFA induced not only peripheral inflammation but also microglial cell activation and increased the expression of proinflammatory cytokines (IL-1 $\beta$, TNF- $\alpha$, and IL- 6 ) in the lumbar spinal cord, brainstem, and forebrain ${ }^{41}$. In addition, differences in cytokine response after CFA injection have been found in the hypothalamus: Charles River rats responded with increased TNF- $\alpha$, IFN- $\gamma$, and IL- 6 production with the development of adjuvant-induced arthritis, whereas Harlan rats had high hypothalamic levels of these 3 cytokines under control conditions ${ }^{42}$. Another recent study using a similar CFA model in mice demonstrated elevated IL-1 $\beta$, IL- 6 , and TNF- $\alpha$ levels in the serum and expression of these pro-inflammatory cytokines in the basolateral amygdala of CFA-injected mice ${ }^{43}$. Also, chronic systemic inflammation promoted neuroinflammation mainly induced by IL- $1 \beta$-positive microglia in female rats ${ }^{44}$. A previous study by our research group showed that CFA-induced orofacial pain increased brainstem IL-6 levels and decreased brainstem IL-10 levels, suggesting that the pain model induced an imbalance in the inflammatory system regarding the structure analyzed ${ }^{45}$. Altogether these studies highlight that peripheral CFA injection induces a response of inflammatory signaling in the peripheral and central nervous system.

This study has some limitations. All female rats underwent ovariectomy, and there was no ovariectomized group without tDCS treatment. Also, all rats were subjected to a model of chronic inflammatory pain. In addition, we did not use a positive control antiinflammatory agent to verify the inflammatory profile. However, some points highlighted above have been discussed in our previous studies ${ }^{18}$.

In summary, based on our findings, bimodal tDCS had an effect on the central inflammatory axis, with a small effect on the peripheral site as evaluated by histology in the current study. However, further studies are encouraged to better understand the relationship of inflammatory diseases in postmenopausal women and the effects of tDCS on these 2 associated conditions.

\section{Funding}

This work was supported by the Graduate Research Group of Hospital de Clínicas de Porto Alegre - GPPG (Grant \#140262).

\section{Conflicts of interest}

The authors report no conflicts of interest. The authors alone are responsible for the content and writing of the paper.

\section{Acknowledgements}

This research was supported by the following Brazilian funding agencies: National Council for Scientific and Technological Development - CNPq (Dr. ILS Torres; Dr. Caumo W; VL Scarabelot); Brazilian Federal Agency for Support and Evaluation of Graduate Education - CAPES (C de Oliveira); Graduate Research Group of Hospital de Clínicas de Porto Alegre - GPPG (ILS Torres - Grant 140262) PIBIC HCPA/CNPq. MCT/FINEP - COENG/2013 and Doc-Fix FAPERGS/CNPq/2012.

\section{REFERENCES}

1. Calvin M. Oestrogens and wound healing. Maturitas. 2000;34(3):195-210.

2. Gomez CR, Plackett TP, Kovacs EJ. Aging and estrogen: modulation of inflammatory responses after injury. Exp Gerontol. 2007;42(5):451-6.

3. Shivers KY, Amador N, Abrams L, Hunter D, Jenab S, QuiñonesJenab V. Estrogen alters baseline and inflammatory-induced cytokine levels independent from hypothalamicpituitary-adrenal axis activity. Cytokine. 2015;72(2):121-9.
4. Conde DM, Verdade RC, Valadares ALR, Mella LFB, Pedro AO, Costa-Paiva L. Menopause and cognitive impairment: A narrative review of current knowledge. World J Psychiatry. 2021;11(8):412-28.

5. Chen BL, Li YQ, Xie DH, He QL, Yang XX. Blocking TNF- $\alpha$ with infliximab alleviates ovariectomy induced mechanical and thermal hyperalgesia in rats. Neurol Sci. 2012;33(3):527-33.

6. Deswal A, Petersen NJ, Feldman AM, Young JB, White BG, Mann DL. Cytokines and cytokine receptors in advanced heart failure: an analysis of the cytokine database from the Vesnarinone Trial (VEST). Circulation. 2001;103(16):2055-9.

7. Priyanka HP, Sharma U, Gopinath S, Sharma V, Hima L, ThyagaRajan S. Menstrual cycle and reproductive aging alters immune reactivity, NGF expression, antioxidant enzyme activities, and intracellular signaling pathways in the peripheral blood mononuclear cells of healthy women. Brain Behav Immun. 2013;32:131-43. 
8. Măluțan AM, Costin N, Ciortea R, Mihu D. Variation of Anti-inflammatory Cytokines in Relationship with Menopause. Appl Med Inform. 2013;32(2):30-8

9. Berchtold NC, Cribbs DH, Coleman PD, Rogers J, Head E, Kim R, et al. Gene expression changes in the course of normal brain aging are sexually dimorphic. Proc Natl Acad Sci U S A. 2008;105(40):15605-10.

10. Sandberg K, Ji H, Einstein G, Au A, Hay M. Is immune system-related hypertension associated with ovarian hormone deficiency? Exp Physiol. 2016;101(3):368-74.

11. Sassarini J, Anderson RA. New pathways in the treatment for menopausal hot flushes. Lancet. 2017;389(10081):1775-7.

12. Archer DF, Sturdee DW, Baber R, de Villiers TJ, Pines A, Freedman RR, et al. Menopausal hot flushes and night sweats: where are we now? Climacteric. 2011;14(5):515-28.

13. Iwasa T, Matsuzaki T, Tungalagsuvd A, Munkhzaya M, Kawami T, Kato T, et al. Effects of ovariectomy on the inflammatory responses of female rats to the central injection of lipopolysaccharide. J Neuroimmunol. 2014;277(1-2):50-6.

14. Gaumond I, Arsenault P, Marchand S. The role of sex hormones on formalininduced nociceptive responses. Brain Res. 2002;958(1):139-45.

15. Kuba T, Hunter D, Zhou L, Jenab S, Quinones-Jenab V. Endogenous gonadal hormones regulate females' behavioral responses to formalin through prostaglandin E2 release. Ethn Dis. 2010;20(1 Suppl 1):S1-55-9.

16. Ceccarelli I, Fiorenzani P, Massafra C, Aloisi AM. Long-term ovariectomy changes formalin-induced licking in female rats: the role of estrogens. Reprod Biol Endocrinol. 2003;1:24.

17. Laste G, Caumo W, Adachi LNS, Rozisky JR, Macedo IC, Marques Filho PR, et al. After-effects of consecutive sessions of transcranial direct current stimulation (tDCS) in a rat model of chronic inflammation. Exp Brain Res. 2012;221(1):75-83.

18. Silva Moreira SF, Medeiros LF, Souza A, Oliveira C, Scarabelot VL, Fregni F, et al. Transcranial direct current stimulation (tDCS) neuromodulatory effects on mechanical hyperalgesia and cortical BDNF levels in ovariectomized rats. Life Sci. 2016;145:233-9.

19. Kilkenny C, Browne WJ, Cuthill IC, Emerson M, Altman DG. Improving bioscience research reporting: the ARRIVE guidelines for reporting animal research. PLoS Biol. 2010;8(6):e1000412.

20. Park SB, Lee YJ, Chung CK. Bone mineral density changes after ovariectomy in rats as an osteopenic model: stepwise description of double dorso-lateral approach. J Korean Neurosurg Soc. 2010;48(4):309-12.

21. Bernardi A, Zilberstein ACCV, Jäger E, Campos MM, Morrone FB, Calixto JB, et al. Effects of indomethacin-loaded nanocapsules in experimental models of inflammation in rats. $\mathrm{Br} \mathrm{J}$ Pharmacol. 2009;158(4):1104-11.

22. Goldman JM, Murr AS, Cooper RL. The rodent estrous cycle: Characterization of vaginal cytology and its utility in toxicological studies. Birth Defects Res B Dev Reprod Toxicol. 2007;80(2):84-97.

23. Lopes BC, Medeiros LF, Silva de Souza V, Cioato SG, Medeiros HR, Regner GG, et al. Transcranial direct current stimulation combined with exercise modulates the inflammatory profile and hyperalgesic response in rats subjected to a neuropathic pain model: Long-term effects. Brain Stimul. 2020;13(3):774-82.

24. Cioato SG, Medeiros LF, Marques Filho PR, Vercelino R, Souza A, Scarabelot VL, et al. Long-Lasting Effect of Transcranial Direct Current Stimulation in the Reversal of Hyperalgesia and Cytokine Alterations Induced by the Neuropathic Pain Model. Brain Stimul. 2016;9(2):209-17.

25. Rueger MA, Keuters MH, Walberer M, Braun R, Klein R, Sparing R, et al. Multi-session transcranial direct current stimulation (tDCS) elicits inflammatory and regenerative processes in the rat brain. PLoS One. 2012;7(8):e43776.

26. Adachi LNS, Caumo W, Laste G, Medeiros LF, Rozisky JR, Souza A, et al. Reversal of chronic stress-induced pain by transcranial direct current stimulation (tDCS) in an animal model. Brain Res. 2012;1489:17-26.
27. Ouyang W, Rutz S, Crellin NK, Valdez PA, Hymowitz SG. Regulation and Functions of the IL-10 Family of Cytokines in Inflammation and Disease. Annu Rev Immunol. 2011;29:71-109.

28. Schunck RVA, Torres ILS, Laste G, Souza A, Macedo IC, Valle MTC, et al. Protracted alcohol abstinence induces analgesia in rats: Possible relationships with BDNF and interleukin-10. Pharmacol Biochem Behav. 2015;135:64-9.

29. Mantyh PW, Koltzenburg M, Mendell LM, Tive L, Shelton DL. Antagonism of nerve growth factorTrkA signaling and the relief of pain. Anesthesiology. 2011;115(1):189-204.

30. McKelvey L, Shorten GD, O'Keeffe GW. Nerve growth factor-mediated regulation of pain signalling and proposed new intervention strategies in clinical pain management. $J$ Neurochem. 2013;124(3):276-89.

31. Chang DS, Hsu E, Hottinger DG, Cohen SP. Anti-nerve growth factor in pain management: current evidence. J Pain Res. 2016;9:373-83.

32. Fritsch B, Reis J, Martinowich $\mathrm{K}$, Schambra HM, Ji Y, Cohen LG, et al. Direct current stimulation promotes BDNF-dependent synaptic plasticity: potential implications for motor learning. Neuron. 2010;66(2):198-204.

33. Brunoni AR, Ferrucci $R$, Bortolomasi M, Vergari M, Tadini L, Boggio PS, et al. Transcranial direct current stimulation (tDCS) in unipolar vs. bipolar depressive disorder. Prog Neuropsychopharmacol Biol Psychiatry. 2011;35(1):96-101.

34. Ruohonen J, Karhu J. tDCS possibly stimulates glial cells. Clin Neurophysiol. 2012;123(10):2006-9.

35. Adachi LNS, Quevedo AS, Souza A Scarabelot VL, Rozisky JR, Oliveira $\mathrm{C}$, et al. Exogenously induced brain activation regulates neuronal activity by top-down modulation: conceptualized model for electrical brain stimulation. Exp Brain Res. 2015;233(5):1377-89.

36. Ribeiro $H$, Sesterhenn RB, Souza A, Souza AC, Alves M, Machado JC, et al. Preoperative transcranial direct current stimulation: Exploration of a novel strategy to enhance neuroplasticity before 
surgery to control postoperative pain. A randomized sham-controlled study. PLoS One. 2017;12(11):e0187013.

37. Zaringhalam J, Manaheji $\mathrm{H}$, Mghsoodi N, Farokhi B, Mirzaiee V. Spinal $\mu$-opioid receptor expression and hyperalgesia with dexamethasone in chronic adjuvant-induced arthritis in rats. Clin Exp Pharmacol Physiol. 2008;35(11):1309-15.

38. Philippe L, Gegout-Pottie P, Guingamp C, Bordji K, Terlain B, Netter $P$, et al. Relations between functional, inflammatory, and degenerative parameters during adjuvant arthritis in rats. Am J Physiol. 1997;273(4):R1550-6.

39. Patil KR, Patil $C R$, Jadhav RB, Mahajan VK, Patil PR, Gaikwad PS. Anti-arthritic activity of bartogenic acid isolated from fruits of Barringtonia racemosa Roxb. (Lecythidaceae). Evidence-based Complement Altern Med. 2011;2011:785245.

40. Spears R, Dees LA, Sapozhnikov M, Bellinger LL, Hutchins B. Temporal changes in inflammatory mediator concentrations in an adjuvant model of temporomandibular joint inflammation. J Orofac Pain. 2005;19(1):34-40.

41. Raghavendra V, Tanga FY, DeLeo JA. Complete Freunds adjuvant-induced peripheral inflammation evokes glial activation and proinflammatory cytokine expression in the CNS. Eur J Neurosci. 2004;20(2):467-73.

42. Bodnar TS, Taves MD, Lavigne KM, Woodward TS, Soma KK, Weinberg J. Differential activation of endocrine-immune networks by arthritis challenge: Insights from colony-specific responses. Sci Rep. 2017;7(1):698.

43. Luo L, Sun $T$, Yang L, Liu $A$, Liu $Q Q$, Tian QQ, et al. Scopoletin ameliorates anxiety-like behaviors in complete Freund's adjuvant-induced mouse model. Mol Brain. 2020;13(1):15.

44. Liu X, Wu Z, Hayashi Y, Nakanishi H. Age-dependent neuroinflammatory responses and deficits in long-term potentiation in the hippocampus during systemic inflammation. Neuroscience. 2012;216:133-42.

45. Scarabelot VL, Oliveira C, Medeiros LF, Macedo IC, Cioato SG, Adachi LNS, et al. Transcranial direct-current stimulation reduces nociceptive behaviour in an orofacial pain model. J Oral Rehabil. 2019;46(1):40-50. 